\title{
Metabolic dysfunction and immunometabolism in COVID-19 pathophysiology and therapeutics
}

\author{
Rachael Batabyal ${ }^{1,2} \cdot$ Nathaniel Freishtat $^{1} \cdot$ Elaise Hill $^{1,2} \cdot$ Muhammad Rehman $^{2} \cdot$ Robert Freishtat $^{1,2} \cdot$ \\ loannis Koutroulis ${ }^{1,2}$
}

Received: 13 September 2020 / Revised: 22 February 2021 / Accepted: 4 March 2021 / Published online: 16 March 2021

(c) The Author(s), under exclusive licence to Springer Nature Limited 2021

\begin{abstract}
The COVID-19 pandemic has emerged as a public health crisis and has placed a significant burden on healthcare systems. Patients with underlying metabolic dysfunction, such as type 2 diabetes mellitus and obesity, are at a higher risk for COVID19 complications, including multi-organ dysfunction, secondary to a deranged immune response, and cellular energy deprivation. These patients are at a baseline state of chronic inflammation associated with increased susceptibility to the severe immune manifestations of COVID-19, which are triggered by the cellular hypoxic environment and cytokine storm. The altered metabolic profile and energy generation of immune cells affect their activation, exacerbating the imbalanced immune response. Key immunometabolic interactions may inform the development of an efficacious treatment for COVID19. Novel therapeutic approaches with repurposed drugs, such as PPAR agonists, or newly developed molecules such as the antagomirs, which block microRNA function, have shown promising results. Those treatments, alone or in combination, target both immune and metabolic pathways and are ideal for septic COVID-19 patients with an underlying metabolic condition.
\end{abstract}

\section{Introduction}

In December of 2019, reports emerged of a cluster of patients with severe pneumonia due to a novel strain of coronavirus (i.e., severe acute respiratory syndrome (SARS)-CoV-2). Many patients infected with SARS-CoV-2 suffered from a severe syndrome called COVID-19, including many typical features of sepsis such as acute respiratory distress syndrome, cardiac injury, and cytokine storm [1, 2]. Observations from early COVID-19 cases pointed to risk factors for mortality, including advanced age and comorbidities common in the elderly but also in

These authors contributed equally: Rachael Batabyal, Nathaniel Freishtat

Ioannis Koutroulis

ikoutrouli@childrensnational.org

1 Division of Emergency Medicine, Children's National Hospital, Washington, DC, USA

2 The George Washington University School of Medicine and Health Sciences, Washington, DC, USA patients with metabolic diseases such as obesity and type 2 diabetes mellitus (T2DM) [3]. In fact, people with obesity and diabetes have an increased susceptibility to severe respiratory complications from SARS-CoV-2 and systemic pathology, such as sepsis [4].

Early data in the United States demonstrated a twofold increase in the need for acute or critical care in patients with obesity, defined as a body mass index (BMI) over 30 (normal BMI $=20-25$ ). This risk increased even further with class 2 or 3 obesity (i.e., a BMI above 35) [4]. One in three patients with obesity infected by SARS-CoV-2, developed respiratory failure leading to invasive mechanical ventilation. Metabolic dysfunction has not only been associated with severe respiratory complications from SARSCoV-2 but also systemic pathology, such as sepsis [5-8].

Obesity and diabetes are associated with increased mortality and long-term sequelae in COVID-19 [1-4, 9, 10]. These conditions are also associated with increased inflammation and disturbance of normal energy production pathways, which can significantly affect the immune response to infection $[3,4,9,11]$. Understanding the interaction between innate immunity and metabolism, termed immunometabolism, can help explain the mechanisms associated with the most severe clinical presentations 
of COVID-19. Herein we will review the latest research on how metabolic dysfunction is associated with chronic inflammation, which leads to a hyperimmune response in these patients upon SARS-CoV-2 infection. We will also discuss therapeutic approaches that address immunometabolic pathways likely to be affected in these patients.

\section{Metabolic dysfunction as a key factor in hyperimmune response and impaired viral clearance of COVID-19}

The increased risks associated with obesity and diabetes are secondary to an imbalance in the immunologic and metabolic pathways. As reviewed by Hotamsligil et al., this delicate balance of the immune system and metabolism has developed over time, allowing the host to fight off infection and avoid starvation [12]. When overnutrition or inflammation tip this balance, they disrupt immune and metabolic homeostasis, leading to a vicious cycle [12]. The effect of obesity and diabetes on immunometabolic pathways favors a pro-inflammatory state and may explain the increased risk of COVID-19 in patients with chronic disease.

The pro-inflammatory state in COVID-19 may be worsened by underlying inflammatory changes associated with insulin resistance [13, 14]. Insulin resistance is one of the hallmark metabolic derangements in metabolically unhealthy obesity and a precursor to T2DM. Inflammatory cells, such as natural killer (NK) cells, appear to be involved in developing insulin resistance [15]. In a high-fat diet mouse model of obesity, NK cell upregulation in visceral adipose tissue was associated with increases in TNF- $\alpha$, interleukin (IL)-6, and IFN- $\gamma$. When NK cells were depleted, insulin sensitivity significantly improved [15]. Others have demonstrated that adipose tissue dendritic cells are also involved in insulin resistance [16]. Taken together, these findings indicate that immune cells affect metabolic pathways, but the relationship between the immune system and metabolism is bidirectional. Adipose tissue and serum in patients with obesity show an upregulation of inflammatory cytokines such as TNF- $\alpha$ IL- 6 , and IL- $1 \beta$, as well as acute phase reactants including $\mathrm{C}$-reactive protein and ferritin [17-19]. Insulin receptor dysfunction contributes to a state of chronic inflammation by activating c-Jun $\mathrm{N}$ terminal kinase and NF-kB. This activation decreases antiinflammatory cytokine production and is associated with dysregulation of inflammatory cell metabolic checks and balances [20-23]. From this, we can conclude that metabolic dysfunction in insulin resistance and obesity is associated with the upregulation of immune cells and cytokines, which contribute to a chronic, subclinical inflammatory process, sometimes termed metaflammation. Metaflammation allows for a hyperimmune response secondary to an infectious insult, including that caused by SARS-CoV-2.

While metabolic dysfunction leads to metaflammation, obesity is a heterogeneous condition, and not all patients with obesity are metabolically unhealthy [24]. Metabolic function seems to be at least partially dependent on the mechanism by which adipocytes expand. Adipose hyperplasia and hypertrophy do not result in metabolically equivalent tissue [25]. The latter is associated with increased inflammation and insulin resistance and an increase in hypoxia-inducible factor 1 alpha (HIF-1 $\alpha$ ), which may explain the susceptibility to severe infections, including COVID-19 [25]. The hyperimmune response to COVID-19 is likely a combination of two distinct pathways: a direct, cytokine-driven activation of systemic inflammation and an indirect, hypoxia-inducible factor (HIF)-driven syndrome [26]. These changes are associated with increased risk of and mortality from sepsis and are negative prognostic factors in COVID-19 [27]. A cascade of pro-inflammatory cytokines regulates the direct pathway, most importantly IL-1 $\beta$ and IL-6. When SARS-COV-2 enters the pulmonary epithelium, toll-like receptors (TLRs) 2,3 , and 4 are activated, and IL-1 $\beta$ and IL- 6 are released. This cytokine release provokes fever, systemic inflammation, and subsequently, sepsis [28]. Obesity is associated with a higher expression of both IL-6 and the IL-6 receptor at baseline. Unlike in lean counterparts, in patients with obesity, the IL-6 receptor is not downregulated by increased cytokine levels, leading to a constant uncontrolled inflammatory state [29].

The indirect pathway is induced by hypoxia and is facilitated by circulating HIFs. These transcription factors become stable under hypoxic conditions and activate several cellular metabolic changes in immune cells [30]. Stable HIFs favor glycolytic metabolism and increased survival of immune cells [30]. The chronic hypoxic conditions of obesity activate HIF- $1 \alpha$ in macrophages, which increases both glycolysis and oxidative phosphorylation and induces M1 macrophage polarization [31]. Therefore, patients with obesity have a propensity for a hyperimmune response secondary to adipocyte hypoxia. This baseline hypoxia, exacerbated by COVID-19 infection, results in inflammation and hypoperfusion that only make cellular hypoxia more severe [32].

Both T2DM and obesity are associated with dysregulation and decreased effector function of both the innate and adaptive immune systems [33]. In murine models of obesity and diabetes, monocytes and neutrophils show reduced phagocytic ability and reduction in the generation of reactive oxygen species $[34,35]$. NK cells in adults with diabetes have a decreased expression of viral ligands and degranulation capabilities [36]. In children, these cells display a decreased cytotoxic killing ability affecting viral 
Fig. 1 Pathophysiologic changes in patients with COVID-19 and metabolic dysfunction and potential therapeutic agents. This graph depicts the mechanisms by which SARS-CoV-2 infection leads to hyperimmune response and sepsis in patients with metabolic dysfunction as well as potential therapeutic molcules with their targets. Diffuse tissue hypoxia and viral replication in the pulmonary epithelium along with TLR activation result in immune cell activation, cytokine release and immunomodulation. SARS-CoV-2 severe acute respiratory syndrome coronavirus 2, IL interleukin, TLR toll-like receptor, HIF hypoxia-inducible factor, PPAR peroxisome proliferatoractivated receptor.

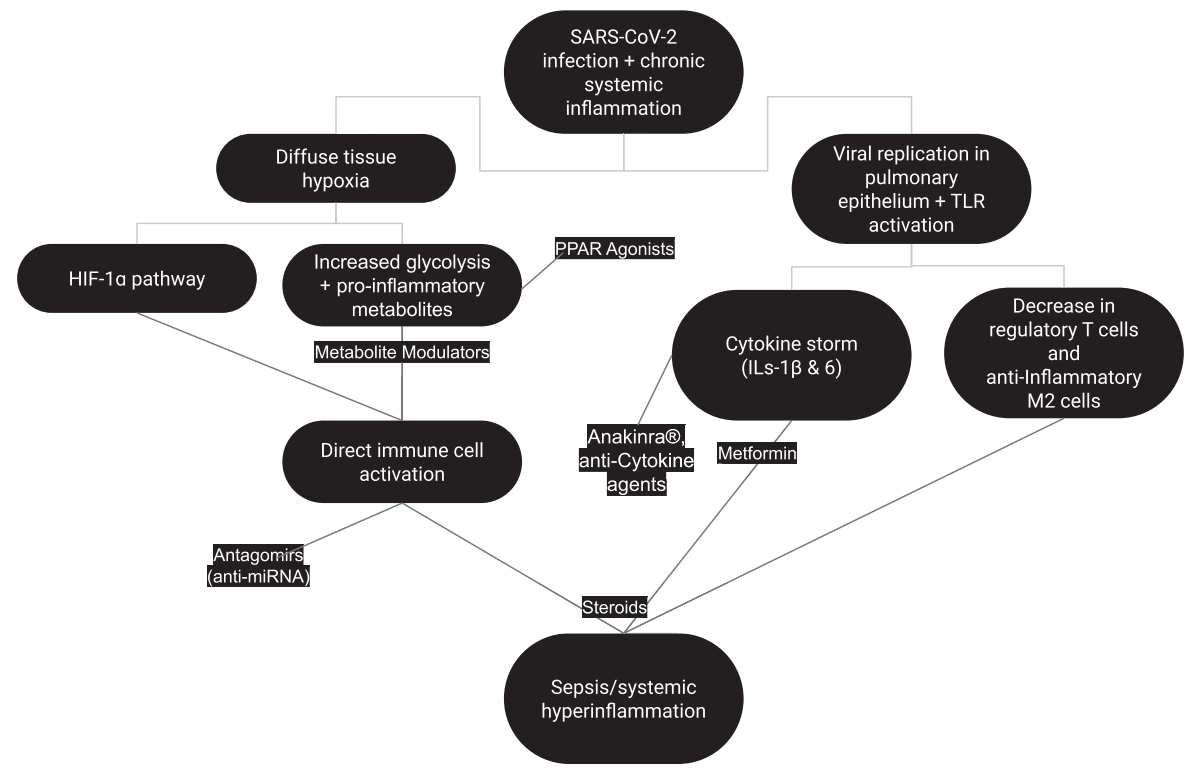

clearance [37]. In addition, type I IFNs directly inhibit viral replication and support the host's immune response against viruses [38]. In preclinical studies of SARS-CoV-2 infection, a delayed IFN- $\alpha$ response in the lungs was associated with increased viral replication, impaired viral clearance, and excessive cytokine release [39]. The downregulation of IFN- $\alpha$ in obesity may impair SARS-COV-2 clearance [40]. Moreover, treatment with IFN- $\alpha$ given to hospitalized patients with SARS-associated coronavirus infection during the 2003 outbreak in Canada, resulted in improvement of lung function [39].

\section{Therapeutic approaches for COVID-19 in patients with metabolic dysfunction}

Obesity and T2DM are very heterogeneous conditions with varying degrees of symptomatology and prognosis [41]. Patients with diabetes may also have different levels of metabolic dysfunction based on adherence to diet and treatment efficacy. Metabolic dysfunction is not always present in patients with obesity. A subset of patients without the underlying pathophysiologic changes expected with an increased BMI is characterized as the "metabolically healthy obese" [24]. At the same time, there are cases of patients with "normal" weights but with increased metabolic risks that should be stratified accordingly [42]. Furthermore, not all metabolically unhealthy patients have the same degree of underlying immunometabolic changes, so an accurate assessment of metabolic health is vital for a mechanistic approach to COVID-19 treatment. A summary of major pathophysiologic changes in patients with COVID-19 and an underlying metabolic dysfunction along with potential therapeutic agents are included in Fig. 1.

\section{Modulation of metabolites can mitigate the hyperimmune response}

The metabolic state of the host is an important factor in the immune response and its regulation. The decrease in the overall number of regulatory $\mathrm{T}$ cells and antiinflammatory M2 macrophages in obesity and T2DM leads to increased secretion of inflammatory cytokines in the adipose tissue and chronic inflammation [43]. The change in macrophage polarization is accompanied by a differential metabolism of M1 and M2 macrophages. M1 macrophages preferentially utilize glycolysis, unlike M2 macrophages, which mainly utilize oxidative phosphorylation and fatty acid oxidation [44]. Interestingly, some studies suggest that the metabolic profile of adipose tissue macrophages (ATM) in obesity is characterized by an upregulation of both glycolysis and oxidative phosphorylation that does not seem to fit either an M1 or M2 predominance. This raises the possibility of a mixed-type metabolism in chronic inflammation secondary to obesity $[45,46]$. Lactate, an end product of glycolysis, promotes macrophage M2-like activity through a HIF-1a mechanism, promoting T-cell trafficking and retention in inflamed tissue [47]. Other metabolites act as feedback regulators to the immunometabolic pathways. For example, itaconate and succinate, both Krebs cycle byproducts, directly activate immune cells [21]. Targeting those metabolites might help develop effective treatments that will alleviate the immunometabolic dysfunction in COVID-19. 
Treatments that target cytokine-driven inflammation show promising results

As new potential therapies for COVID-19 emerge, it is important to consider how the cytokine-driven hyperimmune response can be reversed or mitigated, especially in patients with metabolic dysregulation. Conti et al. suggest the possible benefits of therapy with the newly described antiinflammatory cytokines IL-37 and IL-38, which block the binding of IL-1 $\beta$ and IL- 6 to their receptors [48]. The prospect of anti-cytokine therapy has also prompted a look into existing drugs that target the IL-1 family. More specifically, the immunosuppressant drug Anakinra ${ }^{\circledR}$ (Orphan Biovitrum, Sweden) is already being used off-label to treat severe cases of COVID-19 and multisystem inflammatory syndrome in children secondary to COVID-19. The drug acts by binding to the IL-1 type I receptor, competitively inhibiting action of pro-inflammatory IL-1a and IL- $1 \beta$. With a $95 \%$ bioavailability and a 4-6-h half-life, it is pharmacologically very effective and the first few trials in patients with inflammatory manifestations of COVID-19 have been very promising with $90 \%$ survival rate to the 21 -day mark, compared to $56 \%$ of the control group [49]. Since patients with obesity and T2DM present with a chronic inflammatory state, they might be a target group for Anakinra ${ }^{\odot}$, although more clinical trials are required to prove efficacy.

\section{The role of PPARs and metformin in COVID-19 treatment}

Immunometabolic pathways are modulated in part by the peroxisome proliferator-activated receptors (PPARs). These proteins are members of the nuclear receptor superfamily of transcriptional regulators and essential to glucose and lipid homeostasis [50]. PPAR $\beta / \delta$ and PPAR $\gamma$ play an important role in macrophage polarization [51]. In a mouse model, knockout of PPAR $\gamma$ increased IL- $1 \beta$, IL- 6 , and TNF- $\alpha$ when exposed to lipopolysaccharide [52]. Unlike purely anti-inflammatory treatments, drugs targeting PPARs can have a dual effect on both immune and metabolic pathways [50]. Thiazolidinediones such as rosiglitazone and pioglitazone, both PPAR $\gamma$ agonists, improve cardiac function and overall mortality in animal models of sepsis and decrease the local inflammatory response by lowering IL- $1 \beta$, TNF- $\alpha$, and IL-6 levels [53, 54]. In addition, dual PPAR $\alpha$ and PPAR $\gamma$ agonism with saroglitazar in preclinical models of high-fat diet can restrict weight gain and attenuate inflammatory response. These effects are mediated by M2 macrophages in white adipose tissue and improve overall glucose homeostasis [55]. A recent study of PPAR $\alpha$ agonist, fenofibrate, demonstrated reversal of metabolic changes caused by SARS-cov- 2 and inhibited viral replication in lung epithelial cells [56].
The reliance of immune cells on metabolic pathways for activation is critical to identifying therapeutic targets. At rest, cells favor oxidative phosphorylation via the tricarboxylic acid cycle but revert to glycolytic metabolism in an inflammatory state. The connection between glucose metabolism and immunity in T2DM may explain metformin's therapeutic effect by regulating both pathways. While its primary effect is on glucose metabolism, it also reduces plasma inflammatory marker levels, including TNF- $\alpha$ and IL-6. The effects on TNF- $\alpha$ are more pronounced in females $[57,58]$. In one large observational study, women hospitalized for COVID-19 with obesity or T2DM on metformin therapy had reduced mortality (OR 0.792) compared to those not taking metformin [58].

\section{Steroid use in COVID-19 remains controversial}

Recent reports about the widely used corticosteroid dexamethasone emphasized its ability to inhibit cytokine production and improve mortality of COVD-19-infected patients. However, some authors recommend caution in steroid use for critically ill COVID-19 patients due to the concurrent inhibition of protective antibodies, potentially leading to increased plasma viral load [59, 60]. Current COVID-19 research is not only focused on repurposing approved drugs, but also discovering new therapies targeting the pathophysiologic mechanisms of the disease.

\section{The development of newer COVID-19 therapeutic agents that target immunometabolism}

Recent literature demonstrates that extracellular vesicles, including exosomes, carry miRNA that target immune and metabolic pathways, including those involving the PPARs $[31,61]$. An example of those exosomal microRNAs (miRNA) is miR-155 that is overexpressed in ATM of obese mice and induces insulin resistance. The role of miR-155 was also highlighted in a study in which human adipocytes were treated with TNF- $\alpha$ leading to increased miR-155 expression and inflammation [61]. miR-155 inhibits PPAR $\gamma$ with subsequent activation of the pro-inflammatory NF-kB pathway $[61,62]$. Other miRNAs such as miR-146a/b, miR200b/c, miR-203, and miR-223 have also been implicated in the regulation of the TLR4-induced NF-KB signaling pathway [63-67]. In addition, miR-16, miR-221, miR-579, and miR-125b induce TNF- $\alpha$ expression, favoring a proinflammatory state $[68,69]$. While these microRNAs affect the inflammatory process alone, Let-7 increases IL-6 inflammatory cytokine levels [70] and, at the same time, promotes glucose intolerance and reduced pancreatic insulin secretion [71]. Such miRNAs with a dual role in immunometabolic pathways are potential therapeutic targets in patients with metabolic dysfunction and COVID-19. 
The emergence of anti-miRNA treatments in recent years is based on miRNAs' identifiable regulatory role in several processes, including inflammation [72]. The use of antagomirs, engineered antisense miRNAs that inhibit endogenous miRNAs, can be a valuable tool against the inflammatory dysregulation of metabolic syndromes. Several preclinical studies have demonstrated successful therapeutic miRNA silencing in rheumatoid arthritis and lupus, which are conditions that involve a hyperimmune response [73]. Other miRNAs such as miR-103 and miR-107 regulate insulin sensitivity in vivo, and their respective antagomirs lead to improved glucose homeostasis and insulin sensitivity in murine models of obesity [74]. Despite the positive results of experiments using animal models, further investigation is necessary to identify the mechanisms behind these results and evaluate for potential unwanted effects on endogenous miRNA function. Those treatments could be given alone or in combination with other molecules such as PPAR agonists for enhanced results.

Newer treatments in use for COVID-19 include antibody cocktails, either isolated directly from infected patients or manufactured. Pharmaceutical companies, such as Regeneron and Eli Lilly, are leading the antibody treatment development against COVID-19 (both given emergency authorization by the Food and Drug Administration), while at least ten more antibodies are being tested in trials [75]. Small antibody-like molecules called nanobodies, based on naturally secreted antibodies by camelids, are in development and might be preferred due to their lower cost and easier production process [76]. The potential synergistic effects of combined treatments that both damper inflammation and regulate metabolism may alleviate the hyperimmune response of COVID-19 patients with underlying metabolic dysfunction due to obesity or T2DM.

\section{Conclusions}

Immunometabolic pathways may suggest new treatments for COVID-19. It is vital to recognize that metabolism is a mediator of immunity and the hyperinflammatory state that leads to multi-organ dysfunction in patients with underlying obesity or diabetes. COVID-19 respiratory infection causes a systemic hyperimmune response, which is likely mediated by a shift from oxidative phosphorylation to glycolysis in immune cells. This is triggered by the hypoxic environment due to respiratory failure and the ensuing cytokine storm. Populations at a baseline state of chronic inflammation, such as those with obesity or diabetes, are more susceptible to the severe inflammatory manifestations from COVID-19. Many therapies that alter immunometabolic pathways are currently in clinical trials. The drug Anakinra ${ }^{\circledR}$ has been identified as a potential candidate as it inhibits the IL-1 type
I receptor, and early studies with the drug have been promising. Other treatment approaches target the PPARs, which directly affect the cytokine cascade, inflammatory cell activation, and cellular respiration. Newer therapeutic modalities, including antagomirs and antibody cocktails, alone or in combination with traditional anti-inflammatory drugs such as steroids, present a unique opportunity to prevent the deleterious sequelae of COVID-19 infection in patients with metabolic dysregulation.

\section{Compliance with ethical standards}

Conflict of interest The authors declare no competing interests.

Publisher's note Springer Nature remains neutral with regard to jurisdictional claims in published maps and institutional affiliations.

\section{References}

1. Zhu N, Zhang D, Wang W, Li X, Yang B, Song J, et al. A novel coronavirus from patients with pneumonia in China, 2019. N Engl J Med. 2020;382:727-33.

2. Huang C, Wang Y, Li X, Ren L, Zhao J, Hu Y, et al. Clinical features of patients infected with 2019 novel coronavirus in Wuhan, China. Lancet. 2020;395:497-506.

3. Onder G, Rezza G, Brusaferro S. Case-fatality rate and characteristics of patients dying in relation to COVID-19 in Italy. JAMA. 2020;323:1775-76.

4. Lighter J, Phillips M, Hochman S, Sterling S, Johnson D, Francois $\mathrm{F}$, et al. Obesity in patients younger than 60 years is a risk factor for COVID-19 hospital admission. Clin Infect Dis. 2020;71:896-7.

5. Falagas ME, Kompoti M. Obesity and infection. Lancet Infect Dis. 2006;6:438-46.

6. Chang YC, Huang KT, Chen YM, Wang CC, Wang YH, Tseng $\mathrm{CC}$, et al. Ventilator dependence risk score for the prediction of prolonged mechanical ventilation in patients who survive sepsis/ septic shock with respiratory failure. Sci Rep. 2018;8:5650.

7. Bonafe M, Prattichizzo F, Giuliani A, Storci G, Sabbatinelli J, Olivieri F. Inflamm-aging: why older men are the most susceptible to SARS-CoV-2 complicated outcomes. Cytokine Growth Factor Rev. 2020;53:33-7.

8. Franceschi C, Bonafe M, Valensin S, Olivieri F, De Luca M, Ottaviani E, et al. Inflamm-aging. An evolutionary perspective on immunosenescence. Ann N Y Acad Sci. 2000;908:244-54.

9. Zhou F, Yu T, Du R, Fan G, Liu Y, Liu Z, et al. Clinical course and risk factors for mortality of adult inpatients with COVID-19 in Wuhan, China: a retrospective cohort study. Lancet. 2020; 395:1054-62.

10. Palaiodimos L, Kokkinidis DG, Li W, Karamanis D, Ognibene J, Arora S, et al. Severe obesity, increasing age and male sex are independently associated with worse in-hospital outcomes, and higher in-hospital mortality, in a cohort of patients with COVID19 in the Bronx, New York. Metabolism. 2020;108:154262.

11. Goyal P, Choi JJ, Pinheiro LC, Schenck EJ, Chen R, Jabri A, et al. Clinical characteristics of Covid-19 in New York City. N Engl J Med. 2020;382:2372-4.

12. Hotamisligil GS. Inflammation and metabolic disorders. Nature. 2006;444:860-7.

13. Shimobayashi M, Albert V, Woelnerhanssen B, Frei IC, Weissenberger D, Meyer-Gerspach AC, et al. Insulin resistance causes inflammation in adipose tissue. J Clin Invest. 2018;128:1538-50. 
14. Russo L, Lumeng CN. Properties and functions of adipose tissue macrophages in obesity. Immunology. 2018;155:407-17.

15. Lee BC, Kim MS, Pae M, Yamamoto Y, Eberle D, Shimada T, et al. Adipose natural killer cells regulate adipose tissue macrophages to promote insulin resistance in obesity. Cell Metab. 2016;23:685-98.

16. Cho KW, Zamarron BF, Muir LA, Singer K, Porsche CE, DelProposto JB, et al. Adipose tissue dendritic cells are independent contributors to obesity-induced inflammation and insulin resistance. J Immunol. 2016;197:3650-61.

17. Hotamisligil GS. Foundations of immunometabolism and implications for metabolic health and disease. Immunity. 2017;47:406-20.

18. Bremer AA, Devaraj S, Afify A, Jialal I. Adipose tissue dysregulation in patients with metabolic syndrome. J Clin Endocrinol Metab. 2011;96:E1782-8.

19. Andersen CJ, Murphy KE, Fernandez ML. Impact of obesity and metabolic syndrome on immunity. Adv Nutr. 2016;7:66-75.

20. Weichhart T, Hengstschlager M, Linke M. Regulation of innate immune cell function by mTOR. Nat Rev Immunol. 2015;15:599-614.

21. O'Neill LA, Kishton RJ, Rathmell J. A guide to immunometabolism for immunologists. Nat Rev Immunol. 2016;16:553-65.

22. Norata GD, Caligiuri G, Chavakis T, Matarese G, Netea MG, Nicoletti A, et al. The cellular and molecular basis of translational immunometabolism. Immunity. 2015;43:421-34.

23. Hameed I, Masoodi SR, Mir SA, Nabi M, Ghazanfar K, Ganai BA. Type 2 diabetes mellitus: from a metabolic disorder to an inflammatory condition. World J Diabetes. 2015;6:598-612.

24. Mathew H, Farr OM, Mantzoros CS. Metabolic health and weight: Understanding metabolically unhealthy normal weight or metabolically healthy obese patients. Metabolism. 2016;65:73-80.

25. Shao M, Vishvanath L, Busbuso NC, Hepler C, Shan B, Sharma $\mathrm{AX}$, et al. De novo adipocyte differentiation from Pdgfrbeta $(+)$ preadipocytes protects against pathologic visceral adipose expansion in obesity. Nat Commun. 2018;9:890.

26. Karhausen J, Haase VH, Colgan SP. Inflammatory hypoxia: role of hypoxia-inducible factor. Cell Cycle. 2005;4:256-8.

27. Docherty AB, Harrison EM, Green CA, Hardwick HE, Pius R, Norman L, et al. Features of 16,749 hospitalised UK patients with COVID-19 using the ISARIC WHO Clinical Characterisation Protocol. medRxiv. 2020. https://doi.org/10.1101/2020.04.23. 20076042

28. Conti P, Ronconi G, Caraffa A, Gallenga CE, Ross R, Frydas I, et al. Induction of pro-inflammatory cytokines (IL-1 and IL-6) and lung inflammation by Coronavirus-19 (COVI-19 or SARS-CoV2): anti-inflammatory strategies. J Biol Regul Homeost Agents. 2020;34:327-31.

29. Sindhu S, Thomas R, Shihab P, Sriraman D, Behbehani K, Ahmad R. Obesity is a positive modulator of IL-6R and IL-6 expression in the subcutaneous adipose tissue: significance for metabolic inflammation. PLoS ONE. 2015;10:e0133494.

30. Krzywinska E, Stockmann C. Hypoxia, metabolism and immune cell function. Biomedicines. 2018;6:56.

31. Pan Y, Hui X, Hoo RLC, Ye D, Chan CYC, Feng $T$, et al. Adipocyte-secreted exosomal microRNA-34a inhibits M2 macrophage polarization to promote obesity-induced adipose inflammation. J Clin Invest. 2019;129:834-49.

32. Hosogai N, Fukuhara A, Oshima K, Miyata Y, Tanaka S, Segawa $\mathrm{K}$, et al. Adipose tissue hypoxia in obesity and its impact on adipocytokine dysregulation. Diabetes. 2007;56:901-11.

33. Frydrych LM, Bian G, O'Lone DE, Ward PA, Delano MJ. Obesity and type 2 diabetes mellitus drive immune dysfunction, infection development, and sepsis mortality. J Leukoc Biol. 2018;104:525-34.

34. Frydrych LM, Bian G, Fattahi F, Morris SB, O'Rourke RW, Lumeng CN, et al. GM-CSF administration improves defects in innate immunity and sepsis survival in obese diabetic mice. $\mathbf{J}$ Immunol. 2019;202:931-42.

35. Kuwabara WMT, Yokota CNF, Curi R, Alba-Loureiro TC. Obesity and Type 2 diabetes mellitus induce lipopolysaccharide tolerance in rat neutrophils. Sci Rep. 2018;8:17534.

36. Berrou J, Fougeray S, Venot M, Chardiny V, Gautier JF, Dulphy N, et al. Natural killer cell function, an important target for infection and tumor protection, is impaired in type 2 diabetes. PLoS ONE. 2013;8:e62418.

37. Tobin LM, Mavinkurve M, Carolan E, Kinlen D, O'Brien EC, Little MA, et al. NK cells in childhood obesity are activated, metabolically stressed, and functionally deficient. JCI Insight. 2017;2:e94939.

38. Klionsky DJ, Abdelmohsen K, Abe A, Abedin MJ, Abeliovich H, Acevedo Arozena A, et al. Guidelines for the use and interpretation of assays for monitoring autophagy (3rd edition). Autophagy. 2016;12:1-222.

39. Acharya D, Liu G, Gack MU. Dysregulation of type I interferon responses in COVID-19. Nat Rev Immunol. 2020;20:397-8.

40. Teran-Cabanillas E, Montalvo-Corral M, Caire-Juvera G, Moya-Camarena SY, Hernandez J. Decreased interferon-alpha and interferon-beta production in obesity and expression of suppressor of cytokine signaling. Nutrition. 2013;29:207-12.

41. Neeland IJ, Poirier P, Despres JP. Cardiovascular and metabolic heterogeneity of obesity: clinical challenges and implications for management. Circulation. 2018;137:1391-406.

42. St-Onge MP, Janssen I, Heymsfield SB. Metabolic syndrome in normal-weight Americans: new definition of the metabolically obese, normal-weight individual. Diabetes Care. 2004;27:2222-8.

43. Lumeng CN, Liu J, Geletka L, Delaney C, Delproposto J, Desai A, et al. Aging is associated with an increase in $\mathrm{T}$ cells and inflammatory macrophages in visceral adipose tissue. J Immunol. 2011;187:6208-16.

44. Viola A, Munari F, Sanchez-Rodriguez R, Scolaro T, Castegna A. The metabolic signature of macrophage responses. Front Immunol. 2019;10:1462.

45. Boutens L, Hooiveld GJ, Dhingra S, Cramer RA, Netea MG, Stienstra R. Unique metabolic activation of adipose tissue macrophages in obesity promotes inflammatory responses. Diabetologia. 2018;61:942-53.

46. Sharma M, Boytard L, Hadi T, Koelwyn G, Simon R, Ouimet M, et al. Enhanced glycolysis and HIF-1alpha activation in adipose tissue macrophages sustains local and systemic interleukin-1beta production in obesity. Sci Rep. 2020;10:5555.

47. Soto-Heredero G, Gomez de Las Heras MM, Gabande-Rodriguez E, Oller J, Mittelbrunn M. Glycolysis - a key player in the inflammatory response. FEBS J. 2020;287:3350-69.

48. Dinarello CA. Overview of the IL-1 family in innate inflammation and acquired immunity. Immunol Rev. 2018;281:8-27.

49. Cavalli G, De Luca G, Campochiaro C, Della-Torre E, Ripa M, Canetti D, et al. Interleukin-1 blockade with high-dose anakinra in patients with COVID-19, acute respiratory distress syndrome, and hyperinflammation: a retrospective cohort study. Lancet Rheumatol. 2020;2:e325-e31.

50. Koutroulis I, Batabyal R, McNamara B, Ledda M, Hoptay C, Freishtat RJ. Sepsis immunometabolism: from defining sepsis to understanding how energy production affects immune response. Crit Care Explor. 2019;1:e061.

51. Kang K, Reilly SM, Karabacak V, Gangl MR, Fitzgerald K, Hatano B, et al. Adipocyte-derived Th2 cytokines and myeloid PPARdelta regulate macrophage polarization and insulin sensitivity. Cell Metab. 2008;7:485-95.

52. Silveira LS, Biondo LA, de Souza Teixeira AA, de Lima Junior EA, Castoldi A, Camara NOS, et al. Macrophage immunophenotype but not anti-inflammatory profile is modulated by peroxisome proliferator-activated receptor gamma (PPARgamma) in exercised obese mice. Exerc Immunol Rev. 2020;26:10-22. 
53. Pol CJ, Lieu M, Drosatos K. PPARs: protectors or opponents of myocardial function? PPAR Res. 2015;2015:835985.

54. Kaplan J, Nowell M, Chima R, Zingarelli B. Pioglitazone reduces inflammation through inhibition of NF-kappaB in polymicrobial sepsis. Innate Immun. 2014;20:519-28.

55. Kumar DP, Caffrey R, Marioneaux J, Santhekadur PK, Bhat M, Alonso $\mathrm{C}$, et al. The PPAR alpha/gamma agonist saroglitazar improves insulin resistance and steatohepatitis in a diet induced animal model of nonalcoholic fatty liver disease. Sci Rep. 2020;10:9330.

56. Ehrlich A, Uhl, S, Ioannidis, K, Hofree, M, tenOever, B, Nahmias, Y. The SARS-CoV-2 transcriptional metabolic signature in lung epithelium. Cell Metabolism. 2020.

57. Palsson-McDermott EM, O'Neill LAJ. Targeting immunometabolism as an anti-inflammatory strategy. Cell Res. 2020;30:300-14.

58. Bramante $\mathrm{C}$, Ingraham $\mathrm{N}$, Murray $\mathrm{T}$, Marmor $\mathrm{S}$, Hoversten $\mathrm{S}$, Gronski J, et al. Observational study of metformin and risk of mortality in patients hospitalized with Covid-19. medRxiv. 2020.

59. Group RC, Horby P, Lim WS, Emberson JR, Mafham M, Bell JL, et al. Dexamethasone in hospitalized patients with Covid-19preliminary report. N Engl J Med. 2020.

60. Theoharides TC, Conti P. Dexamethasone for COVID-19? Not so fast. J Biol Regul Homeost Agents. 2020;34:1241-43.

61. Karkeni E, Astier J, Tourniaire F, El Abed M, Romier B, Gouranton $\mathrm{E}$, et al. Obesity-associated inflammation induces microRNA-155 expression in adipocytes and adipose tissue: outcome on adipocyte function. J Clin Endocrinol Metab. 2016;101:1615-26.

62. Li H, Ooi SQ, Heng CK. The role of NF-small ka, CyrillicB in SAA-induced peroxisome proliferator-activated receptor gamma activation. Atherosclerosis. 2013;227:72-8.

63. Ma X, Becker Buscaglia LE, Barker JR, Li Y. MicroRNAs in NFkappaB signaling. J Mol Cell Biol. 2011;3:159-66.

64. Curtale G, Mirolo M, Renzi TA, Rossato M, Bazzoni F, Locati M. Negative regulation of Toll-like receptor 4 signaling by IL-10- dependent microRNA-146b. Proc Natl Acad Sci U S A. 2013;110:11499-504.

65. Haneklaus M, Gerlic M, O’Neill LA, Masters SL. miR-223: infection, inflammation and cancer. J Intern Med. 2013;274:215-26.

66. Wendlandt EB, Graff JW, Gioannini TL, McCaffrey AP, Wilson ME. The role of microRNAs miR-200b and miR-200c in TLR4 signaling and NF-kappaB activation. Innate Immun. 2012;18:846-55.

67. Wei J, Huang X, Zhang Z, Jia W, Zhao Z, Zhang Y, et al. MyD88 as a target of microRNA-203 in regulation of lipopolysaccharide or Bacille Calmette-Guerin induced inflammatory response of macrophage RAW264.7 cells. Mol Immunol. 2013;55:303-9.

68. El Gazzar M, McCall CE. MicroRNAs distinguish translational from transcriptional silencing during endotoxin tolerance. J Biol Chem. 2010;285:20940-51.

69. Jing Q, Huang S, Guth S, Zarubin T, Motoyama A, Chen J, et al. Involvement of microRNA in AU-rich element-mediated mRNA instability. Cell. 2005;120:623-34.

70. Iliopoulos D, Hirsch HA, Struhl K. An epigenetic switch involving NF-kappaB, Lin28, Let-7 MicroRNA, and IL6 links inflammation to cell transformation. Cell. 2009;139:693-706.

71. Frost RJ, Olson EN. Control of glucose homeostasis and insulin sensitivity by the Let-7 family of microRNAs. Proc Natl Acad Sci U S A. 2011;108:21075-80.

72. Contreras J, Rao DS. MicroRNAs in inflammation and immune responses. Leukemia. 2012;26:404-13.

73. Zan H, Tat C, Casali P. MicroRNAs in lupus. Autoimmunity. 2014;47:272-85.

74. Trajkovski M, Hausser J, Soutschek J, Bhat B, Akin A, Zavolan M, et al. MicroRNAs 103 and 107 regulate insulin sensitivity. Nature. 2011;474:649-53.

75. Tantibanchachai C. FDA news release: coronavirus (COVID-19) update: FDA authorizes monoclonal antibodies for treatment of COVID-19. 2020.

76. Ledford $\mathrm{H}$. The race to make COVID antibody therapies more potent. Nature. 2020;587:18. 